Pacific

Journal of

Mathematics

HOPF BIFURCATION IN HIGHER DIMENSIONAL

DIFFERENTIAL SYSTEMS VIA THE AVERAGING METHOD

JAUME LLIBRE AND XIANG ZHANG 


\title{
HOPF BIFURCATION IN HIGHER DIMENSIONAL DIFFERENTIAL SYSTEMS VIA THE AVERAGING METHOD
}

\author{
JAUME LLIBRE AND XIANG ZHANG \\ Dedicated to the memory of Professor Ye Yanqian
}

\begin{abstract}
We study the Hopf bifurcation of $\mathfrak{b}^{3}$ differential systems in $\mathbb{R}^{n}$ showing that $l$ limit cycles can bifurcate from one singularity with eigenvalues $\pm b i$ and $n-2$ zeros with $l \in\left\{0,1, \ldots, 2^{n-3}\right\}$. As far as we know this is the first time that it is proved that the number of limit cycles that can bifurcate in a Hopf bifurcation increases exponentially with the dimension of the space. To prove this result, we use first-order averaging theory. Further, in dimension 4 we characterize the shape and the kind of stability of the bifurcated limit cycles. We apply our results to certain fourth-order differential equations and then to a simplified Marchuk model that describes immune response.
\end{abstract}

\section{Introduction and statement of the main results}

In this work we study the Hopf bifurcation of $\mathscr{C}^{3}$ differential systems in $\mathbb{R}^{n}$ with $n \geq 3$ by using first-order averaging theory. We assume that these systems have a singularity at the origin, whose linear part has eigenvalues $\varepsilon a \pm b i$ and $\varepsilon c_{k}$ for $k=3, \ldots, n$, where $\varepsilon$ is a small parameter. Such systems can be written in the form

$$
\begin{aligned}
& \dot{x}=\varepsilon a x-b y+\sum_{i_{1}+\cdots+i_{n}=2} a_{i_{1} \cdots i_{n}} x^{i_{1}} y^{i_{2}} z_{3}^{i_{3}} \cdots z_{n}^{i_{n}}+\mathscr{A}, \\
& \dot{y}=b x+\varepsilon a y+\sum_{i_{1}+\cdots+i_{n}=2} b_{i_{1} \cdots i_{n}} x^{i_{1}} y^{i_{2}} z_{3}^{i_{3}} \cdots z_{n}^{i_{n}}+\mathscr{B}, \\
& \dot{z}_{k}=\varepsilon c_{k} z_{k}+\sum_{i_{1}+\cdots+i_{n}=2} c_{i_{1} \cdots i_{n}}^{(k)} x^{i_{1}} y^{i_{2}} z_{3}^{i_{3}} \cdots z_{n}^{i_{n}}+\mathscr{C}_{k}, \quad k=3, \ldots, n,
\end{aligned}
$$

where $a_{i_{1} \cdots i_{n}}, b_{i_{1} \cdots i_{n}}, c_{i_{1} \cdots i_{n}}^{(k)}, a, b$ and $c_{k}$ are real parameters, $a b \neq 0$, and $\mathscr{A}, \mathscr{B}$ and $\mathscr{C}_{k}$ are the Lagrange expressions of the error function of third order in the expansion of the functions of the system in Taylor series.

MSC2000: 34C23, 34C29, 37G15.

Keywords: limit cycles, generalized Hopf bifurcation, averaging theory. 
Our first result is on the number of limit cycles which can bifurcate from the origin by using the first-order averaging method.

Theorem 1. There exist $\mathscr{b}^{3}$ systems (1) for which $l \in\left\{0,1, \ldots, 2^{n-3}\right\}$ limit cycles bifurcate from the origin at $\varepsilon=0$, that is, for $\varepsilon$ sufficiently small the system has exactly l limit cycles in a neighborhood of the origin and these limit cycles tend to the origin when $\varepsilon \searrow 0$.

Theorem 1 is proved in Section 3. From the proof of Theorem 1 the next result follows immediately.

Corollary 2. There exist quadratic polynomial differential systems (1) (that is, with $\mathscr{A}=\mathscr{B}=\mathscr{C}_{k}=0$ ) for which $l \in\left\{0,1, \ldots, 2^{n-3}\right\}$ limit cycles bifurcate from the origin at $\varepsilon=0$, that is, for $\varepsilon$ sufficiently small the system has exactly l limit cycles in a neighborhood of the origin and these limit cycles tend to the origin when $\varepsilon \searrow 0$.

The study of limit cycles and averaging theory has a long history; see for instance [Marsden and McCracken 1976; Sanders and Verhulst 1985; Ye et al. 1986; Zhang et al. 1992; Verhulst 1991; Li 2003; Buică and Llibre 2004]. But as far as we know our result is the first one showing that the number of bifurcated limit cycles in a Hopf bifurcation can grow exponentially with the dimension of the system.

For lower dimensional systems we have more precise results than the ones stated in Theorem 1. See [Llibre et al. 2007] for a proof of Theorem 1 in dimension 3 restricted to quadratic polynomial differential systems, and for sufficient conditions for the existence or not of one limit cycle and its kind of stability. In dimension 4 we write the system (1) as

$$
\begin{aligned}
& \dot{x}=\varepsilon a x-b y+\sum_{i+j+k+l=2} a_{i j k l} x^{i} y^{j} z^{k} w^{l}+\mathscr{A}, \\
& \dot{y}=b x+\varepsilon a y+\sum_{i+j+k+l=2} b_{i j k l} x^{i} y^{j} z^{k} w^{l}+\mathscr{B}, \\
& \dot{z}=\quad \varepsilon c z+\sum_{i+j+k+l=2} c_{i j k l} x^{i} y^{j} z^{k} w^{l}+\mathscr{C}, \\
& \dot{w}=\quad \varepsilon d w+\sum_{i+j+k+l=2} d_{i j k l} x^{i} y^{j} z^{k} w^{l}+\mathscr{D},
\end{aligned}
$$

where $a_{i j k l}, b_{i j k l}, c_{i j k l}, d_{i j k l}, a, b, c$ and $d$ are real parameters, $a b \neq 0$, and $\mathscr{A}, \mathscr{B}$, $\mathscr{C}$ and $\mathscr{D}$ are the Lagrange expressions of the error function of third order in the expansion of the functions of the system in Taylor series. Without loss we assume that $b>0$. Set 


$$
\begin{aligned}
& A=K G_{1}-L G_{2}, \\
& B=2 a\left(N_{2} F_{1}-2 N_{3} F_{2}\right)+c F_{1}^{2} G_{2}+d F_{1} F_{2} G_{1}, \\
& C=2 a\left(2 a N_{3}-d F_{1} G_{1}\right), \\
& D=2 a\left(M_{1} F_{1}^{2}+2 M_{2} F_{1} F_{2}+M_{3} F_{2}^{2}\right)+c K F_{1}+d L F_{2},
\end{aligned}
$$

$$
\begin{aligned}
& E=-a\left(2 a M_{2} F_{1}+2 a M_{3} F_{2}+d L\right), \\
& \Lambda=d F_{2} G_{1}-2 a N_{2}, \quad \digamma=c F_{2} G_{2}+2 a N_{1}, \quad \Delta=\Lambda^{2}-8 a N_{3} \digamma, \\
& \Gamma=-8 a^{2} c_{0002} N_{3}^{2}+4 a c F_{2} N_{3}^{2}-2 a c_{0011} N_{3} \Lambda-c_{0002}\left(\Lambda^{2}+\Delta\right) / 2, \\
& \Phi=2 c_{0011} C F_{1}\left(2 a B+C F_{2}\right)-2 c B C F_{1}^{2}-2 c_{0020} C^{2} F_{1}^{2}-2 c_{0002}\left(2 a B+C F_{2}\right)^{2}, \\
& \Psi=4 a\left(\left(c F_{2}-2 a c_{0020}\right) \Lambda^{2}+2 a c_{0011} F_{2} \Lambda \digamma-2 a c_{0002} F_{2}^{2} \digamma^{2}\right),
\end{aligned}
$$

where

$$
\begin{gathered}
F_{1}=a_{1001}+b_{0101}, \quad F_{2}=a_{1010}+b_{0110}, \quad G_{1}=c_{0200}+c_{2000}, \quad G_{2}=d_{0200}+d_{2000}, \\
K=d_{0020} F_{1}^{2}-d_{0011} F_{1} F_{2}+d_{0002} F_{2}^{2}, \quad L=c_{0020} F_{1}^{2}-c_{0011} F_{1} F_{2}+c_{0002} F_{2}^{2}, \\
M_{1}=c_{0020} d_{0011}-c_{0011} d_{0020}, \quad M_{2}=c_{0002} d_{0020}-c_{0020} d_{0002}, \\
M_{3}=c_{0011} d_{0002}-c_{0002} d_{0011},
\end{gathered}
$$$$
N_{1}=d_{0020} G_{1}-c_{0020} G_{2}, \quad N_{2}=d_{0011} G_{1}-c_{0011} G_{2}, \quad N_{3}=d_{0002} G_{1}-c_{0002} G_{2} .
$$

Using these quantities we will be able to control the number of bifurcated limit cycles in the Hopf bifurcation of the system (2) and their kind of stability. Our basic assumptions are

$$
F_{1}^{2}+F_{2}^{2} \neq 0, \quad G_{1}^{2}+G_{2}^{2} \neq 0 .
$$

Because if $F_{1}^{2}+F_{2}^{2}=0$ from the proof of our next theorem we can see that system (2) cannot present a Hopf bifurcation, and if $G_{1}^{2}+G_{2}^{2}=0$ then the system (2) either has no Hopf bifurcation or first-order averaging theory that we are using cannot decide if there is a Hopf bifurcation.

Our results on the Hopf bifurcation of the system (2) are the following.

Theorem 3. Consider a $\mathscr{C}^{3}$ system (2) with $G_{1} \neq 0$.

(a.1) Suppose $F_{1} \neq 0, A \neq 0$, and $B^{2}-4 A C>0$. If $\left.4 A E-D B+D \sqrt{B^{2}-4 A C}\right) F_{1}>0$ or $\left.4 A E-D B-D \sqrt{B^{2}-4 A C}\right) F_{1}>0$, the system has a limit cycle tending to a singular point as $\varepsilon \searrow 0$. We denote this limit cycle by $\Gamma_{1 \varepsilon}$ in the first case and by $\Gamma_{2 \varepsilon}$ in the second. When both inequalities are satisfied, distinct limit cycles $\Gamma_{1 \varepsilon}$ and $\Gamma_{2 \varepsilon}$ exist, tending to different singular points as $\varepsilon \searrow 0$. 
(a.2) Suppose $F_{1} \neq 0, A=0, B \neq 0$ and $\Phi G_{1}>0$. Then the system has a limit cycle $\bar{\Gamma}_{1 \varepsilon}$ tending to the origin as $\varepsilon \searrow 0$.

(a.3) Suppose $F_{1}=0, F_{2} \neq 0, N_{2} \neq 0$, and $\Delta>0$. If

$$
\left(\Gamma-\left(2 a c_{0011} N_{2}+c_{0002} \Lambda\right) \sqrt{\Delta}\right) G_{1}>0
$$

or

$$
\left(\Gamma+\left(2 a c_{0011} N_{2}+c_{0002} \Lambda\right) \sqrt{\Delta}\right) G_{1}>0
$$

the system has a limit cycle tending to a singular point as $\varepsilon \searrow 0$. We denote this limit cycle by $\Gamma_{3 \varepsilon}$ in the first case and by $\Gamma_{4 \varepsilon}$ in the second. When both inequalities are satisfied, distinct limit cycles $\Gamma_{3 \varepsilon}$ and $\Gamma_{4 \varepsilon}$ exist, tending to different singular points as $\varepsilon \searrow 0$.

(a.4) For $F_{1}=0, F_{2} \neq 0$ and $N_{2}=0$, if $\Lambda \neq 0$ and $\Psi G_{1}>0$, the system (2) has a limit cycle $\bar{\Gamma}_{3 \varepsilon}$ tending to the origin as $\varepsilon \searrow 0$.

(b) For $\varepsilon>0$ sufficiently small the limit cycles from statements (a.1)-(a.4) are given by the graphs

$$
\begin{aligned}
& \Gamma_{1 \varepsilon}\left\{\begin{aligned}
r(\theta) & \left.=\varepsilon \sqrt{\left(4 A E-D B+D \sqrt{B^{2}-4 A C}\right) /\left(F_{1} A^{2}\right.}\right)+O\left(\varepsilon^{2}\right), \\
z(\theta) & =\varepsilon\left(B-\sqrt{B^{2}-4 A C}\right) /(2 A)+O\left(\varepsilon^{2}\right), \\
w(\theta) & =-\varepsilon\left(4 a A+F_{2}\left(B-\sqrt{B^{2}-4 A C}\right)\right) /\left(2 A F_{1}\right)+O\left(\varepsilon^{2}\right) ;
\end{aligned}\right. \\
& \Gamma_{2 \varepsilon}\left\{\begin{aligned}
r(\theta) & =\varepsilon \sqrt{\left(4 A E-D B-D \sqrt{B^{2}-4 A C}\right) /\left(F_{1} A^{2}\right)}+O\left(\varepsilon^{2}\right), \\
z(\theta) & =\varepsilon\left(B+\sqrt{B^{2}-4 A C}\right) /(2 A)+O\left(\varepsilon^{2}\right), \\
w(\theta) & =-\varepsilon\left(4 a A+F_{2}\left(B+\sqrt{B^{2}-4 A C}\right)\right) /\left(2 A F_{1}\right)+O\left(\varepsilon^{2}\right) ;
\end{aligned}\right. \\
& \bar{\Gamma}_{1 \varepsilon}\left\{\begin{aligned}
r(\theta) & =\varepsilon \sqrt{\Phi /\left(G_{1} B^{2} F_{1}^{2}\right)}+O\left(\varepsilon^{2}\right) \\
z(\theta) & =\varepsilon C / B+O\left(\varepsilon^{2}\right), \\
w(\theta) & =-\varepsilon\left(2 a B+C F_{2}\right) /\left(B F_{1}\right)+O\left(\varepsilon^{2}\right) ;
\end{aligned}\right. \\
& \Gamma_{3 \varepsilon}\left\{\begin{aligned}
r(\theta) & =\varepsilon \sqrt{\left(\Gamma-\left(2 a c_{0011} N_{2}+c_{0002} \Lambda\right) \sqrt{\Delta}\right) /\left(G_{1} F_{2}^{2} N_{2}^{2}\right)}+O\left(\varepsilon^{2}\right), \\
z(\theta) & =-\varepsilon 2 a / F_{2}+O\left(\varepsilon^{2}\right), \\
w(\theta) & =-\varepsilon(\Lambda+\sqrt{\Delta}) /\left(2 N_{2} F_{2}\right)+O\left(\varepsilon^{2}\right) ;
\end{aligned}\right. \\
& \Gamma_{4 \varepsilon}\left\{\begin{aligned}
r(\theta) & =\varepsilon \sqrt{\left(\Gamma+\left(2 a c_{0011} N_{2}+c_{0002} \Lambda\right) \sqrt{\Delta}\right) /\left(G_{1} F_{2}^{2} N_{2}^{2}\right)}+O\left(\varepsilon^{2}\right), \\
z(\theta) & =-\varepsilon 2 a / F_{2}+O\left(\varepsilon^{2}\right), \\
w(\theta) & =-\varepsilon(\Lambda-\sqrt{\Delta}) /\left(2 N_{2} F_{2}\right)+O\left(\varepsilon^{2}\right) ;
\end{aligned}\right. \\
& \bar{\Gamma}_{3 \varepsilon}\left\{\begin{aligned}
r(\theta) & =\varepsilon \sqrt{\Psi /\left(G_{1} F_{2}^{2} \Lambda^{2}\right)}+O\left(\varepsilon^{2}\right), \\
z(\theta) & =-\varepsilon 2 a / F_{2}+O\left(\varepsilon^{2}\right), \\
w(\theta) & =-\varepsilon 2 a \digamma / \Lambda+O\left(\varepsilon^{2}\right),
\end{aligned}\right.
\end{aligned}
$$


where $\theta \in \mathbb{S}^{1}$ and the coordinates $(r, z, w)$ and $\theta$ are defined at the beginning of Section 4.

For the remaining statements we need the system (2) to be at least $\mathscr{b}^{4}$.

(c.1) For $F_{1}>0$ and $A \neq 0$, the limit cycle $\Gamma_{1 \varepsilon}$ (respectively $\Gamma_{2 \varepsilon}$ ) has at least onedimensional stable (respectively unstable) manifold, and consequently the limit cycle is not a global repeller (respectively attractor). For $F_{1}<0$ and $A \neq 0$ the one-dimensional invariant manifold of the limit cycle has converse stability than for $F_{1}>0$.

(c.2) For $F_{1}>0, A=0$ and $B \neq 0$, the limit cycle $\bar{\Gamma}_{1 \varepsilon}$ has at least one-dimensional stable (respectively unstable) manifold provided that $B>0$ (respectively $B<$ $0)$. For $F_{1}<0$ the one-dimensional invariant manifold of the limit cycle has a different stability than for $F_{1}>0$.

(c.3) For $F_{1}=0, F_{2}>0$ and $N_{2} \neq 0$ the limit cycle $\Gamma_{3 \varepsilon}$ (respectively $\Gamma_{4 \varepsilon}$ ) has at least one-dimensional stable (respectively unstable) manifold. For $F_{1}=0$, $F_{2}<0$ and $N_{2} \neq 0$, the one-dimensional invariant manifold of the limit cycles has a different stability than for $F_{2}>0$.

(c.4) For $F_{1}=0, F_{2} \neq 0, N_{2}=0$ and $\Lambda \neq 0$, the limit cycle $\bar{\Gamma}_{3 \varepsilon}$ has at least one-dimensional stable (respectively unstable) manifold provided that $\Delta>0$ (respectively $\Delta<0$ ).

Theorem 3 will be proved in Section 4.

We note that the quantities defined in (3) depend only on the following 18 parameters: $a, b, c, d, a_{1010}, a_{1001}, b_{0110}, b_{0101}, c_{2000}, c_{0200}, c_{0020}, c_{0011}, c_{0002}$, $d_{2000}, d_{0200}, d_{0020}, d_{0011}, d_{0002}$ of the 44 parameters of the system (2). So the Hopf bifurcation depends only on these 18 parameters.

We remark that in (a.2) we need $B \neq 0$, and in (a.4) we need $\Lambda \neq 0$. Otherwise, as it follows from the proof of Theorem 3 , first-order averaging theory cannot decide on the existence of a Hopf bifurcation.

Other studies on the Hopf bifurcation using averaging theory in a different way than ours have been made by Chow and Mallet-Paret [Marsden and McCracken 1976, Section 4.C]. Their results are more general than the present ones, but ours are more precise and provide also the stability of the bifurcated Hopf limit cycles. A related generalized Hopf bifurcation can be found in [Aguirre and Seibert 1999].

We now consider some applications of our Theorem 3. The first one is on the existence of periodic solutions of the fourth order differential equation

$$
\frac{d^{4} x}{d t^{4}}+p \frac{d^{3} x}{d t^{3}}+q \frac{d^{2} x}{d t^{2}}+k \frac{d x}{d t}+l x=f\left(x, \frac{d x}{d t}, \frac{d^{2} x}{d t^{2}}, \frac{d^{3} x}{d t^{3}}\right)
$$


where $p, q, k, l$ are real parameters satisfying

$$
\begin{array}{ll}
p=-(2 a+c+d) \varepsilon, & q=b^{2}+\left(a^{2}+2 a c+2 a d+c d\right) \varepsilon^{2}, \\
k=-\varepsilon\left(b^{2} c+b^{2} d+\left(a^{2} c+a^{2} d+2 a c d\right) \varepsilon^{2}\right), & l=c d \varepsilon^{2}\left(b^{2}+a^{2} \varepsilon\right),
\end{array}
$$

and $f$ is a $\mathscr{b}^{3}$ function whose first-order partial derivatives vanish at the origin of 4-dimensional space.

Theorem 4. For $0<\varepsilon \ll 1$, the system (4) has an isolated periodic solution in a neighborhood of the trivial solution $x=0$ if one of the following conditions holds:

(i) $b_{4} \neq 0, c b_{4}+d b_{3} \neq 0$ and

$$
\Phi^{*}=a\left(b_{1}+b_{5}\right)\left(c d\left(c b_{4}+d b_{3}\right)-2 a b_{4}^{2}\left(c^{2} b_{10}+c d b_{9}+d^{2} b_{8}\right)\right)>0 ;
$$

(ii) $b_{4}=0, b_{3} \neq 0, d \neq 0$ and

$$
\Psi^{*}=a\left(b_{1}+b_{5}\right)\left(c d^{2} b_{3}-2 a\left(c^{2} b_{3}^{2} b_{10}-c d b_{3} b_{9}-d^{2} b_{8}\right)\right)>0,
$$

where the $b_{i}$ are defined in Section 5.

In Section 5 we will prove Theorem 4 and we will present a more detailed statement on the existence and shape of the periodic solution of (4).

The second application is to a system defined by Marchuk [1980] as a simplified model of an immune system, with no influence from damaged organs but with time delay:

$$
\begin{aligned}
\frac{d X}{d t} & =(\beta-\gamma Z) X, & \frac{d Y}{d t} & =\alpha X Z-\mu_{1}(Y-\delta), \\
\frac{d Z}{d t} & =\rho Y-\left(\mu_{2}+\eta \gamma X\right) Z, & \frac{d W}{d t} & =\sigma X-\mu_{3} W,
\end{aligned}
$$

Theorem 5. There is an open set in parameter space for which the system (5) has at least one limit cycle.

A more detailed statement and a proof of Theorem 5 will be given in Section 6 , where we will present the conditions for the existence and stability of limit cycles coming from a Hopf bifurcation.

\section{The first-order averaging method for periodic orbits}

The aim of this section is to present the first-order averaging method as it was obtained in [Buică and Llibre 2004]. Differentiability of the vector field is not needed. The specific conditions for the existence of a simple isolated zero of the averaged function are given in terms of the Brouwer degree. In fact the Brouwer degree theory is the key point in the proof of this theorem. 
Theorem 6. Consider the following differential system

$$
\dot{x}(t)=\varepsilon f(t, x)+\varepsilon^{2} R(t, x, \varepsilon),
$$

where $f: \mathbb{R} \times D \rightarrow \mathbb{R}^{n}, R: \mathbb{R} \times D \times\left(-\varepsilon_{f}, \varepsilon_{f}\right) \rightarrow \mathbb{R}^{n}$ are continuous functions, $T$ periodic in the first variable, and $D$ is an open subset of $\mathbb{R}^{n}$. Define $f^{0}: D \rightarrow \mathbb{R}^{n}$ as

$$
f^{0}(z)=\frac{1}{T} \int_{0}^{T} f(s, z) d s,
$$

and assume that

(i) $f$ and $R$ are locally Lipschitz with respect to $x$;

(ii) for $b \in D$ with $f^{0}(b)=0$, there exists a neighborhood $V$ of $b$ such that $f^{0}(z) \neq 0$ for all $z \in \bar{V} \backslash\{b\}$ and $d_{B}\left(f^{0}, V, b\right) \neq 0$, (where $d_{B}\left(f^{0}, V, b\right)$ denotes the Brouwer degree of $f^{0}$ in the neighborhood $V$ of $b$ ).

Then, for $|\varepsilon|>0$ sufficiently small, there exists an isolated T-periodic solution $\varphi(\cdot, \varepsilon)$ of the system $(6)$ such that $\varphi(b, 0)=b$.

Here we will need some facts from the proof of Theorem 6. Hypothesis (i) assures the existence and uniqueness of the solution of each initial value problem on the interval $[0, T]$. Hence, for each $z \in D$, it is possible to denote by $x(\cdot, z, \varepsilon)$ the solution of (6) with the initial value $x(0, z, \varepsilon)=z$. We consider also the function $\zeta: D \times\left(-\varepsilon_{f}, \varepsilon_{f}\right) \rightarrow \mathbb{R}^{n}$ defined by

$$
\zeta(z, \varepsilon)=\int_{0}^{T}\left(\varepsilon f(t, x(t, z, \varepsilon))+\varepsilon^{2} R(t, x(t, z, \varepsilon), \varepsilon)\right) d t .
$$

From the proof of Theorem 6 we extract the following facts.

Remark 7. Under the assumptions of Theorem 6 for every $z \in D$ the following relation holds:

$$
x(T, z, \varepsilon)-x(0, z, \varepsilon)=\zeta(z, \varepsilon) .
$$

Moreover the function $\zeta$ can be written in the form

$$
\zeta(z, \varepsilon)=\varepsilon f^{0}(z)+O\left(\varepsilon^{2}\right),
$$

where $f^{0}$ is given by (7) and the symbol $O\left(\varepsilon^{2}\right)$ denotes a bounded function on every compact subset of $D \times\left(-\varepsilon_{f}, \varepsilon_{f}\right)$ multiplied by $\varepsilon^{2}$. Moreover, for $|\varepsilon|$ sufficiently small, $z=\varphi(0, \varepsilon)$ is an isolated zero of $\zeta(\cdot, \varepsilon)$.

Note that from Remark 7 it follows that a zero $z$ of the function $\zeta(z, \varepsilon)$ provides initial conditions for a periodic orbit of the system of period $T$. Consequently the zeros of $f^{0}(z)$ when $f^{0}(z)$ is not identically zero also provides periodic orbits of period $T$. 
For a given system there is the possibility that the function $\zeta$ is not globally differentiable, but the function $f^{0}$ is. In fact, only differentiability in some neighborhood of a fixed isolated zero of $f^{0}$ could be enough. When this is the case, one can use the following remark in order to verify the hypothesis (ii) of Theorem 6.

Remark 8. Let $f^{0}: D \rightarrow \mathbb{R}^{n}$ be a $\mathscr{C}^{1}$ function with $f^{0}(b)=0$, where $D$ is an open subset of $\mathbb{R}^{n}$ and $b \in D$. Whenever $b$ is a simple zero of $f^{0}$ (that is, the Jacobian of $f^{0}$ at $b$ is not zero), then there exists a neighborhood $V$ of $b$ such that $f^{0}(z) \neq 0$ for all $z \in \bar{V} \backslash\{b\}$. Then $d_{B}\left(f^{0}, V, b\right) \in\{-1,1\}$.

The following theorem of [Buică et al. 2007] provides the asymptotic stability of the limit cycles obtained by averaging theory only with the $\mathscr{C}^{1}$ differentiability of $f$ and a Lipschitz assumption on $R$.

Theorem 9. If the function $f$ of (6) is $\mathscr{b}^{1}$ and the function $R$ is Lipschitz in a neighborhood of the limit cycle $\varphi(\cdot, \varepsilon)$ given in Theorem 6 by the simple zero $b$ of $f^{0}$, then for $\varepsilon$ sufficiently small if all the eigenvalues of the Jacobian matrix of $f^{0}$ at $b$ have negative (respectively positive) real part, then the limit cycle $\varphi(\cdot, \varepsilon)$ is asymptotically stable (respectively unstable).

Of course if the function $f$ of (6) is $\mathscr{b}^{2}$ and the function $R$ is $\mathscr{C}^{1}$ then we have better information on the kind of stability of the limit cycle $\varphi(\cdot, \varepsilon)$ given in Theorem 6. A proof of this result can be found in [Verhulst 1991] or in [Guckenheimer and Holmes 1990].

Theorem 10. If the function $f$ of (6) is $\mathscr{b}^{2}$ and the function $R$ is $\mathscr{C}^{1}$ in a neighborhood of a simple zero $b$ of $f^{0}$, then for $\varepsilon$ sufficiently small the stability or instability of the limit cycle $\varphi(\cdot, \varepsilon)$ given in Theorem 6 is given by the stability or instability of the singularity $b$ of the averaged system $\dot{z}=\varepsilon f^{0}(z)$. In fact the singularity $b$ has the stability behavior of the Poincaré map associated to the limit cycle $\varphi(\cdot, \varepsilon)$.

\section{Proof of Theorem 1}

Doing the cylindrical change of coordinates

$$
x=r \cos \theta, \quad y=r \sin \theta, \quad z_{i}=z_{i}, \quad i=3, \ldots, n,
$$

in the region $r>0$, the system (1) becomes

$$
\begin{aligned}
\dot{r} & =\varepsilon a r+\sum\left(a_{i_{1} \cdots i_{n}} \cos \theta+b_{i_{1} \cdots i_{n}} \sin \theta\right)(r \cos \theta)^{i_{1}}(r \sin \theta)^{i_{2}} z_{3}^{i_{3}} \cdots z_{n}^{i_{n}}+O(3), \\
\dot{\theta} & =\frac{1}{r}\left(b r+\sum\left(b_{i_{1} \cdots i_{n}} \cos \theta-a_{i_{1} \cdots i_{n}} \sin \theta\right)(r \cos \theta)^{i_{1}}(r \sin \theta)^{i_{2}} z_{3}^{i_{3}} \cdots z_{n}^{i_{n}}+O(3)\right), \\
\dot{z}_{k} & =\varepsilon c_{k} z_{k}+\sum c_{i_{1} \cdots i_{n}}^{(k)}(r \cos \theta)^{i_{1}}(r \sin \theta)^{i_{2}} z_{3}^{i_{3}} \cdots z_{n}^{i_{n}}+O(3), \quad k=3, \ldots, n,
\end{aligned}
$$

where $O(3)=O_{3}\left(r, z_{3}, \ldots, z_{n}\right)$ and the sums range over $i_{1}+\cdots+i_{n}=2$. 
As usual $\mathbb{Z}_{+}$denotes the set of all nonnegative integers. Taking $a_{00 e_{i j}}=b_{00 e_{i j}}=0$ where $e_{i j} \in \mathbb{Z}_{+}^{n-2}$ has the sum of the entries equal to 2 , it is easy to show that in a suitable small neighborhood of $\left(r, z_{3}, \ldots, z_{n}\right)=(0,0, \ldots, 0)$ we have $\dot{\theta} \neq 0$. Then choosing $\theta$ as the new independent variable, the system (10) becomes, in a neighborhood of $\left(r, z_{3}, \ldots, z_{n}\right)=(0,0, \ldots, 0)$,

$$
\begin{gathered}
\frac{d r}{d \theta}=\frac{r\left(\varepsilon a r+\sum\left(a_{i_{1} \cdots i_{n}} \cos \theta+b_{i_{1} \cdots i_{n}} \sin \theta\right)(r \cos \theta)^{i_{1}}(r \sin \theta)^{i_{2}} z_{3}^{i_{3}} \cdots z_{n}^{i_{n}}+O(3)\right)}{b r+\sum\left(b_{i_{1} \cdots i_{n}} \cos \theta-a_{i_{1} \cdots i_{n}} \sin \theta\right)(r \cos \theta)^{i_{1}}(r \sin \theta)^{i_{2}} z_{3}^{i_{3}} \cdots z_{n}^{i_{n}}+O(3)}, \\
\frac{d z_{k}}{d \theta}=\frac{r\left(\varepsilon c_{k} z_{k}+\sum c_{i_{1} \cdots i_{n}}^{(k)}(r \cos \theta)^{i_{1}}(r \sin \theta)^{i_{2}} z_{3}^{i_{3}} \cdots z_{n}^{i_{n}}+O(3)\right)}{b r+\sum\left(b_{i_{1} \cdots i_{n}} \cos \theta-a_{i_{1} \cdots i_{n}} \sin \theta\right)(r \cos \theta)^{i_{1}}(r \sin \theta)^{i_{2}} z_{3}^{i_{3}} \cdots z_{n}^{i_{n}}+O(3)}, \\
k=3, \ldots, n,
\end{gathered}
$$

where the sums range over $i_{1}+\cdots+i_{n}=2$. Note that this system is periodic of period $2 \pi$ in $\theta$.

To apply the averaging theory of Section 2 in the proof of Theorem 1 we rescale the variables, setting

$$
\left(r, z_{3}, \ldots, z_{n}\right)=\left(\rho \varepsilon, \eta_{3} \varepsilon, \ldots, \eta_{n} \varepsilon\right) .
$$

The system (11) becomes

$$
\begin{aligned}
\frac{d \rho}{d \theta} & =\varepsilon f_{1}\left(\theta, \rho, \eta_{3}, \ldots, \eta_{n}\right)+\varepsilon^{2} g_{1}\left(\theta, \rho, \eta_{3}, \ldots, \eta_{n}, \varepsilon\right), \\
\frac{d \eta_{k}}{d \theta} & =\varepsilon f_{k}\left(\theta, \rho, \eta_{3}, \ldots, \eta_{n}\right)+\varepsilon^{2} g_{k}\left(\theta, \rho, \eta_{3}, \ldots, \eta_{n}, \varepsilon\right), \quad k=3, \ldots, n,
\end{aligned}
$$

where

$$
\begin{aligned}
& f_{1}=\frac{1}{b}\left(a \rho+\sum_{i_{1}+\cdots+i_{n}=2}\left(a_{i_{1} \cdots i_{n}} \cos \theta+b_{i_{1} \cdots i_{n}} \sin \theta\right)(\rho \cos \theta)^{i_{1}}(\rho \sin \theta)^{i_{2}} z_{3}^{i_{3}} \cdots z_{n}^{i_{n}}\right), \\
& f_{k}=\frac{1}{b}\left(c \eta_{k}+\sum_{i_{1}+\cdots+i_{n}=2} c_{i_{1} \cdots i_{n}}^{(k)}(\rho \cos \theta)^{i_{1}}(\rho \sin \theta)^{i_{2}} z_{3}^{i_{3}} \cdots z_{n}^{i_{n}}\right) .
\end{aligned}
$$

The system (13) has the form (6) in the averaging theorem with $x=\left(\rho, \eta_{3}, \ldots, \eta_{n}\right)$, $t=\theta, T=2 \pi$, and

$$
\begin{aligned}
& f\left(\theta, \rho, \eta_{3}, \ldots, \eta_{n}\right) \\
& \quad=\left(f_{1}\left(\theta, \rho, \eta_{3}, \ldots, \eta_{n}\right), f_{3}\left(\theta, \rho, \eta_{3}, \ldots, \eta_{n}\right), \ldots, f_{n}\left(\theta, \rho, \eta_{3}, \ldots, \eta_{n}\right)\right)
\end{aligned}
$$

The averaged system of (13) is

$$
\dot{y}=\varepsilon f^{0}(y), \quad y=\left(\rho, \eta_{3}, \ldots, \eta_{n}\right) \in \Omega,
$$


where $\Omega$ is a suitable neighborhood of the origin $\left(\rho, \eta_{3}, \ldots, \eta_{n}\right)=(0,0, \ldots, 0)$, and

with

$$
f^{0}(y)=\left(f_{1}^{0}(y), f_{3}^{0}(y), \ldots, f_{n}^{0}(y)\right),
$$

$$
f_{i}^{0}(y)=\frac{1}{2 \pi} \int_{0}^{2 \pi} f_{i}\left(\theta, \rho, \eta_{3}, \ldots, \eta_{n}\right) d \theta, \quad i=1,3, \ldots, n .
$$

After some calculations we obtain

$$
\begin{aligned}
& f_{1}^{0}=\frac{1}{2 b} \rho\left(2 a+\sum_{j=3}^{n}\left(a_{10 e_{j}}+b_{01 e_{j}}\right) \eta_{j}\right), \\
& f_{k}^{0}=\frac{1}{2 b}\left(2 c_{k} \eta_{k}+\left(c_{20 \mathbf{0}_{n-2}}^{(k)}+c_{02 \mathbf{0}_{n-2}}^{(k)}\right) \rho^{2}+2 \sum_{3 \leq i \leq j \leq n} c_{00 e_{i j}}^{(k)} \eta_{i} \eta_{j}\right), \quad k=3, \ldots, n,
\end{aligned}
$$

where $e_{j} \in \mathbb{Z}_{+}^{n-2}$ is the unit vector with the $j$-th entry equal to 1 , and $e_{i j} \in \mathbb{Z}_{+}^{n-2}$ has the sum of the $i$-th and $j$-th entries equal to 2 and the other equal to 0 .

Now we shall apply Theorem 6 for obtaining limit cycles of the system (13). After the rescaling (12), these limits will become infinitesimal limit cycles for the system (11), which will tend to origin when $\varepsilon \searrow 0$, consequently they will be bifurcated limit cycles of the Hopf bifurcation of the system (11) at the origin.

Using Theorem 6 for studying the limit cycles of the system (13) we only need to compute the nondegenerate singularities of the system (14). Since the transformation from the cartesian coordinates $\left(r, z_{3}, \ldots, z_{n}\right)$ to the cylindrical ones $\left(\rho, \eta_{3}, \ldots, \eta_{n}\right)$ is not a diffeomorphism at $\rho=0$, we deal with the zeros having the coordinate $\rho>0$ of the averaged function $f^{0}$. So we need to compute the roots of the algebraic equations

$$
\begin{aligned}
& 2 a+\sum_{j=3}^{n}\left(a_{10 e_{j}}+b_{01 e_{j}}\right) \eta_{j}=0, \\
& 2 c_{k} \eta_{k}+\left(c_{20 \mathbf{0}_{n-2}}^{(k)}+c_{02 \mathbf{0}_{n-2}}^{(k)}\right) \rho^{2}+2 \sum_{3 \leq i \leq j \leq n} c_{00 e_{i j}}^{(k)} \eta_{i} \eta_{j}=0, k=3, \ldots, n .
\end{aligned}
$$

Since the coefficients of this system are independent and arbitrary, we can simplify the notation by writing the system as

$$
\begin{aligned}
& a+\sum_{j=3}^{n} a_{j} \eta_{j}=0, \\
& c_{0}^{(k)} \rho^{2}+c_{k} \eta_{k}+\sum_{3 \leq i \leq j \leq n} c_{i j}^{(k)} \eta_{i} \eta_{j}=0, \quad k=3, \ldots, n,
\end{aligned}
$$

where $a_{j}, c_{0}^{(k)}, c_{k}$ and $c_{i j}^{(k)}$ are arbitrary constants. 
Denote by $\mathscr{C}$ the set of algebraic systems of form (16). We claim that there is a system belonging to $\mathscr{C}$ which has exactly $2^{n-3}$ simple roots. The claim can be verified by the example

$$
\begin{aligned}
& a+a_{3} \eta_{3}=0, \\
& c_{0}^{(3)} \rho^{2}+c_{3} \eta_{3}+\sum_{3 \leq i \leq j \leq n} c_{i j}^{(3)} \eta_{i} \eta_{j}=0, \\
& c_{k} \eta_{k}+\sum_{3 \leq i \leq j \leq k} c_{i j}^{(k)} \eta_{i} \eta_{j}=0, \quad k=4, \ldots, n,
\end{aligned}
$$

with all the coefficients nonzero. Equations (19) can be treated as quadratic algebraic equations in $\eta_{k}$. Substituting the unique solution $\eta_{30}$ of $\eta_{3}$ in (17) into (19) with $k=4$, then this last equation has exactly two different solutions $\eta_{41}$ and $\eta_{42}$ for $\eta_{4}$ choosing conveniently $c_{4}$. Introducing the two solutions $\left(\eta_{30}, \eta_{4 i}\right), i=1,2$, into (19) with $k=5$ and choosing conveniently the values of the coefficients of (19) with $k=5$ and $\left(\eta_{3}, \eta_{4}\right)=\left(\eta_{30}, \eta_{4 i}\right)$ we get two different solutions $\eta_{5 i 1}$ and $\eta_{5 i 2}$ of $\eta_{5}$ for each $i$. Moreover playing with the coefficients of the equations, the four solutions $\left(\eta_{30}, \eta_{4 i}, \eta_{5 i j}\right)$ for $i, j=1,2$, are distinct. By induction we can prove that for suitable choice of the coefficients equations (17) and (19) have $2^{n-3}$ different roots $\left(\eta_{3}, \ldots, \eta_{n}\right)$. Since $\eta_{3}=\eta_{30}$ is fixed, for any given $c_{i j}^{(3)}$ there exist values of $c_{3}$ and $c_{0}^{(3)}$ such that (18) has a positive solution $\rho$ for each of the $2^{n-3}$ solutions $\left(\eta_{3}, \ldots, \eta_{n}\right)$ of (17) and (19). Since the $2^{n-3}$ solutions are different, and the number of the solutions of (17)-(19) is the maximum that the equations can have by Bézout's Theorem, it follows that every solution is simple, and consequently the determinant of the Jacobian of the system evaluated at it is not zero. This proves the claim.

Using the same arguments which allow us to prove the claim, we also can prove that we can choose the coefficients of the previous system in order that it has $0,1, \ldots, 2^{n-3}-1$ simple real solutions.

Taking the averaged system (14) with $f^{0}$ having the convenient coefficients as in (17)-(19), the averaged system (14) has exactly $k \in\left\{0,1, \ldots, 2^{n-3}\right\}$ singularities with the components $\rho>0$. Moreover the determinants of the Jacobian matrix $\partial f^{0} / \partial y$ at these singularities do not vanish, because all the singularities are simple. By Theorem 6 and Section 2 we get that there are systems of form (1) which have $k \in\left\{0,1, \ldots, 2^{n-3}\right\}$ limit cycles. This proves Theorem 1 .

\section{Proof of Theorem 3}

Following the proof of Theorem 1 after the change of variables $x=r \cos \theta, y=$ $r \sin \theta, z=z$ and $w=w$, and the rescaling $(r, z, w)=(\rho \varepsilon, \xi \varepsilon, \eta \varepsilon)$ we get from 
(2) that

$$
\begin{aligned}
& \frac{d \rho}{d \theta}=\varepsilon A_{1}(\theta, \rho, \xi, \eta)+\varepsilon^{2} B_{1}(\theta, \rho, \xi, \eta, \varepsilon), \\
& \frac{d \xi}{d \theta}=\varepsilon A_{2}(\theta, \rho, \xi, \eta)+\varepsilon^{2} B_{2}(\theta, \rho, \xi, \eta, \varepsilon), \\
& \frac{d \rho}{d \theta}=\varepsilon A_{3}(\theta, \rho, \xi, \eta)+\varepsilon^{2} B_{3}(\theta, \rho, \xi, \eta, \varepsilon),
\end{aligned}
$$

where the $A_{i}$ can be got from the proof of Theorem 1. We do not present them here. The averaged system associated to (20) is

$$
\dot{y}=\varepsilon f^{0}(y), \quad y=(\rho, \xi, \eta),
$$

where $f^{0}(y)=\left(f_{1}^{0}(y), f_{2}^{0}(y), f_{3}^{0}(y)\right)$ with

$$
\begin{aligned}
& f_{1}^{0}=\frac{1}{2 b} \rho\left(2 a+\left(a_{1010}+b_{0110}\right) \xi+\left(a_{1001}+b_{0101}\right) \eta\right), \\
& f_{2}^{0}=\frac{1}{2 b}\left(2 c \xi+\left(c_{0200}+c_{2000}\right) \rho^{2}+2\left(c_{0020} \xi^{2}+c_{0011} \xi \eta+c_{0002} \eta^{2}\right)\right), \\
& f_{3}^{0}=\frac{1}{2 b}\left(2 d \eta+\left(d_{0200}+d_{2000}\right) \rho^{2}+2\left(d_{0020} \xi^{2}+d_{0011} \xi \eta+d_{0002} \eta^{2}\right)\right) .
\end{aligned}
$$

As explained in the proof of Theorem 1, we consider the singularities with $\rho>0$ of the averaged system (21). Some tedious calculations show that for $F_{1} \neq 0$ and $A \neq 0$ the singularities with $\rho>0$ of (21) are $S_{1}=\left(\rho_{1}, \xi_{1}, \eta_{1}\right)$ with

$$
\begin{gathered}
\rho_{1}=\sqrt{\frac{4 A E-D B+D \sqrt{B^{2}-4 A C}}{F_{1} A^{2}}}, \quad \xi_{1}=\frac{B-\sqrt{B^{2}-4 A C}}{2 A}, \\
\eta_{1}=-\frac{4 a A+F_{2}\left(B-\sqrt{B^{2}-4 A C}\right)}{2 A F_{1}},
\end{gathered}
$$

if $B^{2}-4 A C>0$ and $\left(4 A E-D B+D \sqrt{B^{2}-4 A C}\right) F_{1}>0$;

or $S_{2}=\left(\rho_{2}, \xi_{2}, \eta_{2}\right)$ with

$$
\begin{gathered}
\rho_{2}=\sqrt{\frac{4 A E-D B-D \sqrt{B^{2}-4 A C}}{F_{1} A^{2}}}, \quad \xi_{2}=\frac{B+\sqrt{B^{2}-4 A C}}{2 A}, \\
\eta_{2}=-\frac{4 a A+F_{2}\left(B+\sqrt{B^{2}-4 A C}\right)}{2 A F_{1}},
\end{gathered}
$$

if $B^{2}-4 A C>0$ and $\left(4 A E-D B-D \sqrt{B^{2}-4 A C}\right) F_{1}>0$.

We treat $A, B, C, D, E$ as polynomials in $F_{i}$ and $G_{i}$ for $i=1,2$. Then $A E$ has the terms with degree 4 , but $D B$ and $D \sqrt{B^{2}-4 A C}$ has the terms with the lowest degree 5. So by choosing the values of $F_{1}, F_{2}, G_{1}$ and $G_{2}$ suitably small, that is, the values of $a_{1001}, a_{1010}, b_{0110}, b_{0101}, c_{0200}, c_{2000}, d_{0200}, d_{2000}$, and the 
convenient choice of the other parameters we can prove that $S_{1}$ and $S_{2}$ can appear simultaneously.

From other tedious calculations we get that

$$
\left.\operatorname{det}\left(\frac{\partial f^{0}}{\partial y}\right)\right|_{S_{1}}=-\rho_{1}^{2} \frac{\sqrt{B^{2}-4 A C}}{2 b^{3} F_{1}} \text { and }\left.\operatorname{det}\left(\frac{\partial f^{0}}{\partial y}\right)\right|_{S_{2}}=\rho_{2}^{2} \frac{\sqrt{B^{2}-4 A C}}{2 b^{3} F_{1}}
$$

Hence it follows from the assumptions of statement (a.1) of Theorem 3 and the previous discussions that $S_{1}$ and $S_{2}$ can appear simultaneously and are different, and that the determinants of the Jacobian matrix of (21) at $S_{1}$ and $S_{2}$ are both different from zero. By Section 2 we obtain that for $\varepsilon$ sufficiently small the system (20) has two limit cycles that we denoted by $\Gamma_{i \varepsilon}, i=1,2$, and $\Gamma_{i \varepsilon} \rightarrow S_{i}, i=1,2$, as $\varepsilon \searrow 0$. Hence statement (a.1) of Theorem 3 is proved.

If $F_{1} \neq 0, A=0$ and $B \neq 0$, the averaged system (21) has the unique singularity

$$
\bar{S}_{1}=\left(\bar{\rho}_{1}, \bar{\xi}_{1}, \bar{\eta}_{1}\right)=\left(\sqrt{\frac{\Phi}{G_{1} B^{2} F_{1}^{2}}}, \frac{C}{B},-\frac{2 a B+C F_{2}}{B F_{1}}\right) .
$$

Recall that we have assumed $G_{1} \neq 0$. The determinant of the Jacobian matrix of (21) at $\bar{S}_{1}$ is

$$
\left.\operatorname{det}\left(\frac{\partial f^{0}}{\partial y}\right)\right|_{\bar{S}_{1}}=-\bar{\rho}_{1}^{2} \frac{B}{2 b^{3} F_{1}} .
$$

Therefore using Section 2 it follows statement (a.2) of Theorem 3.

If $F_{1}=0, F_{2} \neq 0$ and $N_{2} \neq 0$, the averaged system (21) has the singularities

$$
S_{3}=\left(\rho_{3}, \xi_{3}, \eta_{3}\right)=\left(\sqrt{\frac{\Gamma-\left(2 a c_{0011} N_{2}+c_{0002} \Lambda\right) \sqrt{\Delta}}{G_{1} F_{2}^{2} N_{2}^{2}}},-\frac{2 a}{F_{2}},-\frac{\Lambda+\sqrt{\Delta}}{2 N_{2} F_{2}}\right),
$$

if $\left(\Gamma-\left(2 a c_{0011} N_{2}+c_{0002} \Lambda\right) \sqrt{\Delta}\right) G_{1}>0$; and

$$
S_{4}=\left(\rho_{4}, \xi_{4}, \eta_{4}\right)=\left(\sqrt{\frac{\Gamma+\left(2 a c_{0011} N_{2}+c_{0002} \Lambda\right) \sqrt{\Delta}}{G_{1} F_{2}^{2} N_{2}^{2}}},-\frac{2 a}{F_{2}},-\frac{\Lambda-\sqrt{\Delta}}{2 N_{2} F_{2}}\right),
$$

if $\left(\Gamma+\left(2 a c_{0011} N_{2}+c_{0002} \Lambda\right) \sqrt{\Delta}\right) G_{1}>0$. The determinants of the Jacobian matrix of (21) at $S_{3}$ and $S_{4}$ are respectively

$$
\left.\operatorname{det}\left(\frac{\partial f^{0}}{\partial y}\right)\right|_{S_{3}}=-\rho_{3}^{2} \frac{\sqrt{\Delta F_{2}^{2}}}{2 b^{3} F_{2}} \quad \text { and }\left.\quad \operatorname{det}\left(\frac{\partial f^{0}}{\partial y}\right)\right|_{S_{4}}=\rho_{4}^{2} \frac{\sqrt{\Delta F_{2}^{2}}}{2 b^{3} F_{2}} .
$$

Again from Section 2 it follows statement (a.3) of Theorem 3. 
If $F_{1}=0, F_{2} \neq 0, N_{2}=0$ and $\Lambda \neq 0$, the averaged system (21) has the unique singularity

$$
\bar{S}_{3}:=\left(\bar{\rho}_{3}, \bar{\xi}_{3}, \bar{\eta}_{3}\right)=\left(\sqrt{\frac{\Psi}{G_{1} F_{2}^{2} \Lambda^{2}}},-\frac{2 a}{F_{2}},-\frac{2 a \digamma}{\Lambda}\right) .
$$

The determinant of the Jacobian matrix of (21) at $\bar{S}_{3}$ is

$$
\left.\operatorname{det}\left(\frac{\partial f^{0}}{\partial y}\right)\right|_{\bar{S}_{3}}=-\bar{\rho}_{3}^{2} \frac{\Lambda}{2 b^{3}}
$$

After a similar treating as those in the proof of the case $S_{1}$ and $S_{2}$, we can finished the proof of statement (a.4).

For proving statement (b) of Theorem 3 we observe that the limit cycles $\Gamma_{i \varepsilon}$ for $i=1,2$ can be written in the form $\left\{\left(r_{i}(\theta), z_{i}(\theta), w_{i}(\theta)\right) ; \theta \in \mathbb{S}^{1}\right\}$, and that the singularities $S_{1}$ and $S_{2}$ in the coordinates $(r, z, w)$ are respectively

$$
\begin{aligned}
& \left(\varepsilon \sqrt{\frac{4 A E-D B+D \sqrt{B^{2}-4 A C}}{F_{1} A^{2}}}, \varepsilon \frac{B-\sqrt{B^{2}-4 A C}}{2 A},\right. \\
& \left(\varepsilon \sqrt{\frac{4 A E-D B-D \sqrt{B^{2}-4 A C}}{F_{1} A^{2}}}, \quad-\varepsilon \frac{4 a A+F_{2}\left(B-\sqrt{B^{2}-4 A C}\right)}{2 A F_{1}}\right), \\
& \left.\varepsilon \frac{B+\sqrt{B^{2}-4 A C}}{2 A},-\varepsilon \frac{4 a A+F_{2}\left(B+\sqrt{B^{2}-4 A C}\right)}{2 A F_{1}}\right) .
\end{aligned}
$$

Now the proof of statement (b) of Theorem 3 for the limit cycles $\Gamma_{i \varepsilon}$ with $i=1,2$ follows from Section 2.

In a similar way as for the limit cycles $\Gamma_{1 \varepsilon}$ and $\Gamma_{2 \varepsilon}$ we can get the conclusion for the other limit cycles $\bar{\Gamma}_{1 \varepsilon}, \Gamma_{3 \varepsilon}$ and $\Gamma_{4 \varepsilon}$ and $\bar{\Gamma}_{3 \varepsilon}$. Hence statement (b) of Theorem 3 is proved.

By Theorem 10 and the determinants (22) (respectively (23), (24) and (25)) of the Jacobian matrix of (21) at $S_{1}$ and $S_{2}$ (respectively $\bar{S}_{3}, S_{3}$ and $S_{4}$, and $\bar{S}_{3}$ ), the proof of statements (c.1)-(c.4) of Theorem 3 easily follows.

We remark that the characteristic equations of the system (21) at $S_{i}, i=1,2,3,4$, are extremely complicated in the expression. So we do not pursue further analysis on them to obtain more information on the dimensions of stable and unstable manifolds of the limit cycles $\Gamma_{i \varepsilon}, i=1,2,3,4$. 


\section{Application to fourth-order differential equations}

Now we want to apply our Theorem 3 to study the existence of periodic solutions of higher order differential equations. We consider the following fourth order differential equation

$$
\frac{d^{4} x}{d t^{4}}+p \frac{d^{3} x}{d t^{3}}+q \frac{d^{2} x}{d t^{2}}+k \frac{d x}{d t}+l x=f\left(x, \frac{d x}{d t}, \frac{d^{2} x}{d t^{2}}, \frac{d^{3} x}{d t^{3}}\right),
$$

where $p, q, k, l$ are real parameters, and $f$ is a $b^{3}$ function with the expansion

$$
\begin{aligned}
f\left(x, \frac{d x}{d t}, \frac{d^{2} x}{d t^{2}}, \frac{d^{3} x}{d t^{3}}\right)=a_{1} x^{2}+a_{2} x \frac{d x}{d t} & +a_{3} x \frac{d^{2} x}{d t^{2}}+a_{4} x \frac{d^{3} x}{d t^{3}}+a_{5}\left(\frac{d x}{d t}\right)^{2} \\
& +a_{6} \frac{d x}{d t} \frac{d^{2} x}{d t^{2}}+a_{7} \frac{d x}{d t} \frac{d^{3} x}{d t^{3}}+a_{8}\left(\frac{d^{2} x}{d t^{2}}\right)^{2} \\
& +a_{9} \frac{d^{2} x}{d t^{2}} \frac{d^{3} x}{d t^{3}}+a_{10}\left(\frac{d^{3} x}{d t^{3}}\right)^{2}+O(3) .
\end{aligned}
$$

We assume

$$
\begin{array}{ll}
p=-(2 a+c+d) \varepsilon, & q=b^{2}+\left(a^{2}+2 a c+2 a d+c d\right) \varepsilon^{2}, \\
k=-\varepsilon\left(b^{2} c+b^{2} d+\left(a^{2} c+a^{2} d+2 a c d\right) \varepsilon^{2}\right), & l=c d \varepsilon^{2}\left(b^{2}+a^{2} \varepsilon\right) .
\end{array}
$$

Set $x_{1}=x, x_{2}=\dot{x}_{1}, x_{3}=\dot{x}_{2}, x_{4}=\dot{x}_{3}$ and the dot denotes derivative with respect to the time $t$. Equation (26) can be written as the system

$$
\begin{aligned}
& \dot{x}_{1}=x_{2}, \quad \dot{x}_{2}=x_{3}, \quad \dot{x}_{3}=x_{4}, \\
& x_{4}=-l x_{1}-k x_{2}-q x_{3}-p x_{4}+f\left(x_{1}, x_{2}, x_{3}, x_{4}\right) .
\end{aligned}
$$

Applying the change of variables

$$
(x, y, z, w)^{T}=M\left(x_{1}, x_{2}, x_{3}, x_{4}\right)^{T},
$$

with

$$
M=\left(\begin{array}{cccc}
-a c d \varepsilon^{3} & a c \varepsilon^{2}+a d \varepsilon^{2}+c d \varepsilon^{2} & -a \varepsilon-c \varepsilon-d \varepsilon & 1 \\
b c d \varepsilon^{2} & -b c \varepsilon-b d \varepsilon & b & 0 \\
-d \varepsilon\left(b^{2}+a^{2} \varepsilon^{2}\right) & b^{2}+a^{2} \varepsilon^{2}+2 a d \varepsilon^{2} & -2 a \varepsilon-d \varepsilon & 1 \\
-c \varepsilon\left(b^{2}+a^{2} \varepsilon^{2}\right) & b^{2}+a^{2} \varepsilon^{2}+2 a c \varepsilon^{2} & -2 a \varepsilon-c \varepsilon & 1
\end{array}\right)
$$

the system (27) becomes

$$
\begin{array}{ll}
\dot{x}=\varepsilon a x-b y+g(x, y, z, w), & \dot{y}=b x+\varepsilon a y, \\
\dot{z}=\varepsilon c z+g(x, y, z, w), & \dot{w}=\varepsilon d w+g(x, y, z, w),
\end{array}
$$

where $g(x, y, z, w)=f\left(M^{-1}(x, y, z, w)^{T}\right)$. 


\section{Write}

$$
\begin{aligned}
& g(x, y, z, w) \\
& =b_{1} x^{2}+b_{2} x y+b_{3} x z+b_{4} x w+b_{5} y^{2}+b_{6} y z+b_{7} y w+b_{8} z^{2}+b_{9} z w+b_{10} w^{2}+O(3) .
\end{aligned}
$$

The coefficients of $g$ are functions depending on the entries of the matrix $M$ and on the coefficients of $f$, which we do not give here explicitly. Now for the system (29) the relevant quantities in (3) become

$$
\begin{aligned}
F_{1} & =b_{4}, \quad F_{2}=b_{3}, \quad G_{1}=G_{2}=b_{1}+b_{5}, \quad M_{i}=N_{i}=0, \quad i=1,2,3, \\
A & =0, \quad B=\left(c b_{4}^{2}+d b_{3} b_{4}\right)\left(b_{1}+b_{5}\right), \quad C=-2 a d b_{4}\left(b_{1}+b_{5}\right), \\
\Lambda & =d b_{3}\left(b_{1}+b_{5}\right), \quad \digamma=c b_{3}\left(b_{1}+b_{5}\right), \\
\Phi & =4 a b_{4}^{2}\left(b_{1}+b_{5}\right)^{2}\left(c d\left(c b_{4}+d b_{3}\right)-2 a b_{4}^{2}\left(c^{2} b_{10}+c d b_{9}+d^{2} b_{8}\right)\right), \\
\Psi & =4 a b_{3}^{2}\left(b_{1}+b_{5}\right)^{2}\left(c d^{2} b_{3}-2 a\left(c^{2} b_{3}^{2} b_{10}-c d b_{3} b_{9}+d^{2} b_{8}\right)\right) .
\end{aligned}
$$

Since $A=0$ and $N_{2}=0$, applying Theorem 3 we get the next result.

Corollary 11. Suppose $0<\varepsilon \ll 1$.

(a) System (26) has an isolated periodic solution in a neighborhood of the trivial solution $x=0$ if one of the following conditions holds:

(i) $b_{4} \neq 0, c b_{4}+d b_{3} \neq 0$ and

$$
\Phi^{*}=a\left(b_{1}+b_{5}\right)\left(c d\left(c b_{4}+d b_{3}\right)-2 a b_{4}^{2}\left(c^{2} b_{10}+c d b_{9}+d^{2} b_{8}\right)\right)>0 ;
$$

(ii) $b_{4}=0, b_{3} \neq 0, d \neq 0$ and

$$
\Psi^{*}=a\left(b_{1}+b_{5}\right)\left(c d^{2} b_{3}-2 a\left(c^{2} b_{3}^{2} b_{10}-c d b_{3} b_{9}+d^{2} b_{8}\right)\right)>0 .
$$

(b) The periodic solution has, under condition (i), the asymptotic expression

$$
x=\frac{-2 a d}{(c-d)\left(c b_{4}+d b_{5}\right)\left(b^{2}+(a-c)^{2} \varepsilon^{2}\right)}+\frac{2 a b_{4}\left(c b_{4}+d b_{3}\right)\left(b_{1}+b_{5}\right)+c b_{3}}{(c-d)\left(b^{2}+(a-d)^{2} \varepsilon^{2}\right)}+O(\varepsilon),
$$

and under condition (ii) the asymptotic expression

$$
x=\frac{-2 a}{(c-d) b_{3}\left(b^{2}+(a-c)^{2} \varepsilon^{2}\right)}+\frac{2 a c}{(c-d) d\left(b^{2}+(a-d)^{2} \varepsilon^{2}\right)}+O(\varepsilon) .
$$

(c) The isolated periodic solution is a limit cycle.

The proof of Corollary 11 follows from Theorem 3 and the change of coordinates (9) and (28). The following examples show that do there exist systems of form (26) satisfying the conditions of Corollary 11. 
Example 1. The system

$$
\frac{d^{4} x}{d t^{4}}-5 \varepsilon \frac{d^{3} x}{d t^{3}}+\left(1+9 \varepsilon^{2}\right) \frac{d^{2} x}{d t^{2}}-\varepsilon\left(3+7 \varepsilon^{2}\right) \frac{d x}{d t}+2 \varepsilon^{2}(1+\varepsilon) x=x \frac{d x}{d t}+\frac{7}{6} x \frac{d^{3} x}{d t^{3}}
$$

satisfies condition (i) of Corollary 11 for $\varepsilon$ sufficiently small. Because the equality $b_{4}=-1 /(6 \varepsilon)+O(1)$ holds, we have that $c b_{4}+d b_{3}=-1 /(6 \varepsilon)+O(\varepsilon)$, and $\Phi^{*}=$ $45 / 324+O\left(\varepsilon^{2}\right)$. So it has an isolated periodic solution in a neighborhood of $x=0$.

Example 2. The system

$$
\begin{aligned}
\frac{d^{4} x}{d t^{4}}-2 \varepsilon \frac{d^{3} x}{d t^{3}}+\frac{d^{2} x}{d t^{2}} & +2 \varepsilon^{3} \frac{d x}{d t}-(1+\varepsilon) \varepsilon^{2} x \\
& =-x^{2}+\mu x \frac{d x}{d t}-2 x \frac{d^{2} x}{d t^{2}}+\mu x \frac{d^{3} x}{d t^{3}}+2\left(\frac{d x}{d t}\right)^{2}-\frac{d x}{d t} \frac{d^{2} x}{d t^{2}}
\end{aligned}
$$

satisfies condition (ii) of Corollary 11 for $\mu$ and $\varepsilon$ sufficiently small. As $b_{4}=0$, $b_{3}=-4+O(\varepsilon)$ (or $-4+O\left(\varepsilon^{2}\right)$ if $\left.\mu=0\right), d=1$ and $\Psi^{*}=69 /\left(2 \varepsilon^{2}\right)+O(1 / \varepsilon)$ (or $69 /\left(2 \varepsilon^{2}\right)+O(1)$ if $\left.\mu=0\right)$. So it has an isolated periodic solution in a neighborhood of $x=0$.

\section{Application to the Marchuk system of immune response}

The system

$$
\begin{array}{llrl}
\frac{d V}{d t} & =(\beta-\gamma F) V, & \frac{d C}{d t} & =\alpha V(t-\tau) F(t-\tau)-\mu_{c}(C-\bar{C}), \\
\frac{d F}{d t} & =\rho C-\left(\mu_{f}+\eta \gamma V\right) F, & \frac{d m}{d t} & =\sigma V-\mu_{m} m,
\end{array}
$$

was introduced by Marchuk [1980] as a simplified model of an immune system with no influence from damaged organs. $\bar{C}$ is a constant level of plasma cells in a healthy organism, and the biological meaning of the coordinates and coefficients is given in [Marchuk 1980; Marzeniuk and Nakonechny 2003]. For practical meaning we assume that none of the coefficients vanish. In [Marchuk 1980; Martsenyuk 2004] the authors studied the stability of the equilibrium states.

We will apply our Theorem 3 to the system (30) without the time delay for studying the existence of the periodic solutions. Thus we consider system (5). It has two singularities

$$
\begin{aligned}
& P=\left(0, \delta, \frac{\delta \rho}{\mu_{2}}, 0\right), \\
& Q=\left(\frac{\mu_{1}\left(\beta \mu_{2}-\delta \gamma \rho\right)}{\beta\left(\alpha \rho-\eta \gamma \mu_{1}\right)}, \frac{\alpha \beta \mu_{2}-\delta \eta \gamma^{2} \mu_{1}}{\gamma\left(\alpha \rho-\eta \gamma \mu_{1}\right)}, \frac{\beta}{\gamma}, \frac{\mu_{1} \sigma\left(\beta \mu_{2}-\delta \gamma \rho\right)}{\beta \mu_{3}\left(\alpha \rho-\eta \gamma \mu_{1}\right)}\right) .
\end{aligned}
$$


The first has eigenvalues $-\mu_{1},-\mu_{2},-\mu_{3}, \beta-\delta \gamma \rho / \mu_{2}$. Theorem 3 cannot be applied to it. We consider the possible appearance of limit cycles of the system (5) in a neighborhood of the singularity $Q$. We denote its coordinates by $\left(x^{*}, y^{*}, z^{*}, w^{*}\right)$.

For the system (5) at $Q$ to have the form (2), we choose the parameters as

$$
\begin{aligned}
\mu_{3} & =-d \varepsilon, \\
\mu_{2} & =\frac{b^{2} \beta-\beta^{2} \mu_{1}-\left(2 a \beta^{2}+b^{2} c+\beta^{2} c\right) \varepsilon+m_{2} \beta \varepsilon^{2}-a^{2} c \varepsilon^{3}}{\beta\left(\beta+\mu_{1}\right)}, \\
\eta & =\frac{\alpha\left(b^{2} \beta+\beta \mu_{1}^{2}+m_{1} \varepsilon+m_{2} \beta \varepsilon^{2}-a^{2} c \varepsilon^{3}\right) \rho}{\gamma \beta\left(c \varepsilon+\mu_{1}\right)\left(b^{2}+a^{2} \varepsilon^{2}+2 a \mu_{1} \varepsilon+\mu_{1}^{2}\right)}, \\
\rho & =\frac{\beta\left(b^{2} \mu_{1}-\beta \mu_{1}^{2}-m_{1} \varepsilon+m_{2} \mu_{1} \varepsilon^{2}+a^{2} c \varepsilon^{3}\right)}{\delta \gamma \mu_{1}\left(\beta+\mu_{1}\right)},
\end{aligned}
$$

where $m_{1}=\left(2 a \beta \mu_{1}+c \beta \mu_{1}-b^{2} c\right), m_{2}=\left(a^{2}+2 a c\right)$, and $a, b, c$ and $d$ are the coefficients of the linear part of the system (2), which can be chosen arbitrarily but with $a b \neq 0$. We remark that the choice of $\mu_{3}$ follows from the fact that $-\mu_{3}$ is an eigenvalue of $Q$. We take the coordinate translation

$$
x_{1}=X-x^{*}, \quad x_{2}=Y-y^{*}, \quad x_{3}=Z-z^{*}, \quad x_{4}=W-w^{*},
$$

and an invertible linear change of coordinates $(x, y, z, w)^{T}=M\left(x_{1}, x_{2}, x_{3}, x_{4}\right)^{T}$, where $T$ denotes the transpose of a matrix. The system (5) then becomes

$$
\begin{array}{llrl}
\frac{d x}{d t} & =\varepsilon a x-b y+v_{1} G(x, y, z, w), & \frac{d y}{d t} & =b x+\varepsilon a y+v_{2} G(x, y, z, w), \\
\frac{d z}{d t} & =\varepsilon c z+v_{3} G(x, y, z, w), & \frac{d w}{d t} & =\varepsilon w+v_{4} G(x, y, z, w),
\end{array}
$$

where, setting $\mathcal{N}=\beta \delta \mu_{1}\left(c \varepsilon+\mu_{1}\right)\left(\beta+\mu_{1}\right)^{2}\left(b^{2}+\left(a \varepsilon+\mu_{1}\right)^{2}\right)\left(b^{2}+(a-c)^{2} \epsilon^{2}\right)^{2}$, we have defined

$$
\begin{aligned}
v_{1}= & \frac{1}{b \mathcal{N}}\left(b^{2}\left(\beta^{2}-a \beta \varepsilon+c \varepsilon\left(a \varepsilon+\mu_{1}\right)\right)+(\beta-a \varepsilon)\left(a \varepsilon+\mu_{1}\right)\left(a \varepsilon(\beta-c \varepsilon)+\beta\left(c \varepsilon+\mu_{1}\right)\right)\right), \\
v_{2}= & \frac{1}{\mathcal{N}}\left(-\beta\left(b^{2}+\left(\mu_{1}+a \varepsilon\right)^{2}\right)+c \varepsilon\left(b^{2}+(\beta-a \varepsilon)^{2}\right)\right), \\
v_{3}= & \frac{1}{\mathcal{N}}(c \varepsilon-\beta)\left(b^{2}-\beta \mu_{1}+a \varepsilon(a \varepsilon-2 \beta)\right), \\
v_{4}= & \frac{1}{\mathcal{N}}\left((c \varepsilon-\beta)\left(b^{2}-2 a \beta \varepsilon+a^{2} \varepsilon^{2}\right)-d \beta \mu_{1} \varepsilon+\beta^{2}\left(\mu_{1}+c \varepsilon-d \varepsilon\right)\right), \\
G= & b^{2} c \varepsilon x^{2}+\left(b^{2}+(a-c) a \varepsilon^{2}\right)(a-c) \varepsilon y^{2}+c \varepsilon\left(c \varepsilon+\mu_{1}\right)^{2} z^{2} \\
& \quad-\left(b^{3}+a^{2} b \varepsilon^{2}-b c^{2} \varepsilon^{3}\right) x y-2 b c \varepsilon\left(c \varepsilon+\mu_{1}\right) x z+\left(b^{2}+\left(a^{2}-c^{2}\right) \varepsilon^{2}\right)\left(c \varepsilon+\mu_{1}\right) y z .
\end{aligned}
$$


The expression of the matrix $M=\left(a_{i j}\right)$ is extremely long, we do not present it here. We got the matrix $M$ with mathematica via the choices of $m_{22}=0$, and $m_{23}=m_{33}=m_{43}=\delta \gamma \mu_{1}\left(\beta+\mu_{1}\right)\left(c \varepsilon+\mu_{1}\right)\left(b^{2}+\left(a \varepsilon+\mu_{1}\right)^{2}\right)$.

For the system (32) all the parameters in (3) other than $\Lambda, \digamma, \Delta, F_{2}, G_{1}$ and $G_{2}$ vanish. Moreover we have $\Lambda=d F_{2} G_{1}, \Delta=\Lambda^{2}$,

$$
\begin{gathered}
F_{2}=\frac{1}{\mathcal{N}_{1}}\left(-\left(b^{2}+\mu_{1}^{2}\right) b^{2} \beta+\left(b^{2} c\left(b^{2}-\beta^{2}\right)-2 \beta \mu_{1}\left(a b^{2}+c \beta \mu_{1}\right)\right) \varepsilon\right. \\
+\left(\left(c^{2}-2 a^{2}\right) b^{2} \beta-\mu_{1}\left(2 b^{2} c^{2}+2 c \beta^{2}(c+2 a)+\beta \mu_{1} \ell\right)\right) \varepsilon^{2} \\
+\left(b^{2} c\left(a^{2}+\ell\right)-c \beta^{2}\left(2 a^{2}-\ell\right)-2 a \beta \mu_{1}\left(\ell-2 c^{2}\right)\right) \varepsilon^{3} \\
\left.-\left(a \beta\left(a^{3}-5 a c^{2}-2 c^{3}\right)+2 a^{2} c^{2} \mu_{1}\right) \varepsilon^{4}+a^{2} c \ell \varepsilon^{5}\right)
\end{gathered}
$$

where $\ell=a^{2}-2 a c-c^{2}$ and

$$
\mathcal{N}_{1}=\beta \delta \mu_{1}\left(b^{2}+(a-c)^{2} \varepsilon^{2}\right)^{2}\left(\beta+\mu_{1}\right)^{2}\left(b^{2}+\left(a \varepsilon+\mu_{1}\right)^{2}\right) .
$$

Corollary 12. For $0<\varepsilon \ll 1$, if the parameters of the system (5) satisfy (31), $b^{2}-\beta \mu_{1} \neq 0$ and $a c<0$, then it has a limit cycle in a vicinity of the singularity $Q$. Moreover the limit cycle has three dimensional unstable (respectively stable) manifolds if $c>0$ and $d>0$ (respectively $c<0$ and $d<0$ ), or two dimensional unstable (respectively stable) and one dimensional stable (respectively unstable) manifolds if $c>0$ and $d<0$ (respectively $c<0$ and $d>0$ ).

Proof. The first statement follows from Theorem 3 and the expressions of $F_{2}, G_{1}$, $\Lambda$ and $\Psi G_{1}$.

For proving the second statement, we denote the six coefficients of the polynomial $G$ by $a_{1}, \ldots, a_{6}$. Then the averaged system (21) becomes

$$
\begin{aligned}
& \frac{d \rho}{d \theta}=\frac{1}{2 b} \rho\left(2 a+\left(v_{1} a_{5}+v_{2} a_{6}\right) \xi\right), \\
& \frac{d \xi}{d \theta}=\frac{1}{2 b}\left(2 c \xi+v_{3}\left(a_{2}+a_{1}\right) \rho^{2}+2 v_{3} a_{3} \xi^{2}\right), \\
& \frac{d \eta}{d \theta}=\frac{1}{2 b}\left(2 d \eta+v_{4}\left(a_{2}+a_{1}\right) \rho^{2}+2 v_{4} a_{3} \xi^{2}\right) .
\end{aligned}
$$


It has a unique singularity with $\rho>0$, denoted by $S$. System (33) at $S$ has the eigenvalues

$$
\lambda_{1}=\frac{d}{b}, \quad \lambda_{23}=\frac{E_{b} \pm \sqrt{E_{b}^{2}-4 E_{a} E_{c}}}{2 E_{a}},
$$

where $E_{a}=b^{2} a_{5} v_{1}+b^{2} a_{6} v_{2}, E_{b}=-4 a b a_{3} v_{3}+b c a_{5} v_{1}+b c a_{6} v_{2}$ and $E_{c}=$ $4 a^{2} a_{3} v_{3}-2 a c a_{5} v_{1}-2 a c a_{6} v_{2}$. Direct calculations show that

$$
E_{a} E_{c}=\frac{-2 a c}{b^{2} \delta^{2} \mu_{1}^{2}\left(\beta+\mu_{1}\right)^{2}}+O(\epsilon), \quad E_{a} E_{b}=\frac{c}{b \delta^{2} \mu_{1}^{2}\left(\beta+\mu_{1}\right)^{2}}+O(\epsilon) .
$$

So the second statement of the corollary follows easily from the eigenvalues of $S$ via Theorem 10. This proves the corollary.

\section{Acknowledgements}

The first author is partially supported by a MCYT/FEDER grant number MTM 2008-03437 and by a CICYT grant number 2005SGR 00550. The second author is partially supported by NNSF of China grant 10671123 and 10831003 and NCET of China grant 050391 . He thanks the Centre de Recerca Matemàtica for the hospitality and the financial support grant SAB2006-0098 (Ministerio de Educación y Ciencia, Spain).

\section{References}

[Aguirre and Seibert 1999] L. Aguirre and P. Seibert, "Types of change of stability and corresponding types of bifurcations", Discrete Contin. Dynam. Systems 5:4 (1999), 741-752. MR 2000i:37074 Zbl 0986.37042

[Buică and Llibre 2004] A. Buică and J. Llibre, "Averaging methods for finding periodic orbits via Brouwer degree”, Bull. Sci. Math. 128:1 (2004), 7-22. MR 2004j:34102 Zbl 1055.34086

[Buică et al. 2007] A. Buică, J. Llibre, and O. Makarenkov, "Asymptotic stability of periodic solutions for nonsmooth differential equations with application to the nonsmooth van der Pol oscillator", Preprint, 2007.

[Guckenheimer and Holmes 1990] J. Guckenheimer and P. Holmes, Nonlinear oscillations, dynamical systems, and bifurcations of vector fields, Applied Math. Sciences 42, Springer, New York, 1990. MR 93e:58046 Zbl 0515.34001

[Li 2003] J. Li, "Hilbert's 16-th problem and bifurcations of planar polynomial vector fields", Internat. J. Bifur. Chaos Appl. Sci. Engrg. 13:1 (2003), 47-106. MR 2003k:34002 Zbl 1063.34026

[Llibre et al. 2007] J. Llibre, C. A. Buzzi, and P. R. da. Silva, "3-dimensional Hopf bifurcation via averaging theory", Discrete Contin. Dyn. Syst. 17:3 (2007), 529-540. MR 2007k:34141 Zbl 1137.37026

[Marchuk 1980] G. I. Marchuk, Matematicheskie modeli v immunologii, Nauka, Moscow, 1980. MR 83a:92008 Zbl 0505.92006

[Marsden and McCracken 1976] J. E. Marsden and M. McCracken, The Hopf bifurcation and its applications, Applied Math. Sciences 19, Springer, New York, 1976. MR 58 \#13209 Zbl 0346.58007 
[Martsenyuk 2004] V. P. Martsenyuk, "On stability in an immune protection model taking into account damage to the target organ: the degenerate Lyapunov functional method", Kibernet. Sistem. Anal. 1 (2004), 153-164, 191. In Russian; translated in Cybernet. Systems Anal. 40:1 (2004), 126136. MR 2005f:92031 Zbl 1067.92040

[Marzeniuk and Nakonechny 2003] V. P. Marzeniuk and A. G. Nakonechny, System analysis methods of medical and biological processes, Ukrmedknyha, Ternopil, Ukraine, 2003.

[Sanders and Verhulst 1985] J. A. Sanders and F. Verhulst, Averaging methods in nonlinear dynamical systems, Applied Math. Sciences 59, Springer, New York, 1985. MR 87d:34065 Zbl 0586.34040

[Verhulst 1991] F. Verhulst, Nonlinear differential equations and dynamical systems, Springer, Berlin, 1991. MR 97g:34003 Zbl 0854.34002

[Ye et al. 1986] Y. Q. Ye, S. L. Cai, L. S. Chen, K. C. Huang, D. J. Luo, Z. E. Ma, E. N. Wang, M. S. Wang, and X. A. Yang, Theory of limit cycles, 2nd ed., Translations of Math. Monogr. 66, Amer. Math. Soc., Providence, RI, 1986. MR 88e:58080

[Zhang et al. 1992] Z. F. Zhang, T. R. Ding, W. Z. Huang, and Z. X. Dong, Qualitative theory of differential equations, Translations of Math. Monogr. 101, Amer. Math. Soc., Providence, RI, 1992. MR 93h:34002 Zbl 0779.34001

Received November 18, 2007.

\author{
JAUME LLIBRE \\ DEPARTAMENT DE MATEMÀTIQUeS \\ UNIVERSITAT AUTÒNOMA DE BARCELONA \\ 08193 BELLATERRA, BARCELONA \\ CATALUNYA \\ SPAIN \\ jllibre@mat.uab.cat
}

XIANG Zhang

DEPARTMENT OF MATHEMATICS

SHANGHAi JiAOTONG UNIVERSITY

SHANGHAI 200240

CHINA

xzhang@sjtu.edu.cn 